\title{
A CORRELATION BETWEEN THE DISTRIBUTION OF NITROGEN CENTERS IN DIAMONDS AND THEIR INTERNAL STRUCTURE AND MECHANISM OF GROWTH
}

Khachatryan GK and Kaminsky* FV

Two essential mechanisms of crystal growth are known for natural diamonds: tangential and normal. Tangential (layer-by-layer) growth, following the octahedral scheme, can be treated as evidence of equilibrium conditions of diamond formation. Normal growth is quite the reverse and indicates that crystallization proceeded in a system oversaturated by carbon, and with high growth rates, i.e., under nonequilibrium conditions. In addition, natural diamond frequently exhibits a combined mechanism of growth, usually exhibited in the sectorial structure of diamond crystals. The combined growth mechanism also points to non-equilibrium crystallization conditions. We studied the volumetric distribution of nitrogen centers in several tens of diamond crystals from Yakutia, Arkhangelsk and the Urals. The examined diamonds differed from each other both in morphology (octahedra, dodecahedroids, tetrahexahedroids and cubic crystals) and in internal structure. Among the octahedral diamonds are some crystals with zoned (with closed layers) and zonal-sectorial structures with cub-octahedral cores, and octahedral outer zones. The dodecahedroids are related to the tangential and combined mechanisms of crystal growth by homogeneous, layer-by-layer-step-like zonal, zonal-sectorial and sectorial structures. The sectorial structure is also common in tetrahexahedroids. The cubic diamonds predominantly have a fibrous (columnar) structure, reflecting normal crystal growth. Diamonds with homogeneous, zonal and layer-bylayer-step-like internal structures are characterized by a quite uniform distribution of nitrogen centers and identical temperatures of formation. This is in complete agreement with the 'annealing' conception of nitrogen impurity aggregation in diamonds. However, diamonds with zonal-sectorial, sectorial and fibrous structures show a strongly heterogeneous volumetric distribution of nitrogen centers. The majority of such crystals are characterized by core-to-margin decreases in total nitrogen structural impurity content and by formation temperatures of their outer zones some $\sim 15$ to $45 \mathrm{oC}$ higher than crystallization temperatures of their cores, in same crystal. This is anomalous. Even providing that the temperature increased during crystallization, formation temperatures, of the outermost zone of a diamond crystal, plays the role of annealing temperature for all the internal ones. Hence, the outermost zone of a crystal cannot have a higher formation temperature than the internal crystal zones. We conclude that the distribution of nitrogen impurity centers in diamond crystals with tangential structural zoning, due to slow crystallization, can be considered as equilibrium, reflecting the actual temperature mode of diamond growth. Unlike these diamonds, diamond crystals with normal or combined mechanism of growth are characterized by a high crystallization rate, probably higher than the rate of aggregation of nitrogen atoms in the structure of diamond crystals. Therefore, in diamonds of this type, the concentrations of nitrogen B-centers are nonequilibrium and, accordingly, diamond-formation temperature values are anomalous. The emperature values calculated from IR data do not necessarily reflect the actual growth temperature, as this depends on diamond-forming kinetics. 